Proceedings of the 8th International Symposium on

\title{
FOUNDATIONS OF
}

\section{OUANTUM MECHANICS}

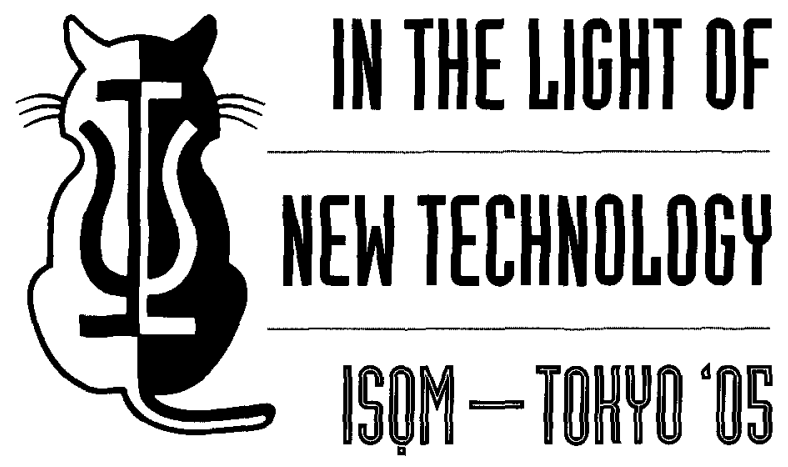


This page is intentionally left blank 
Proceedings of the 8th International Symposium on

\section{FOUNDATIONS OF}

\section{OUAANTUM MECHANICS}

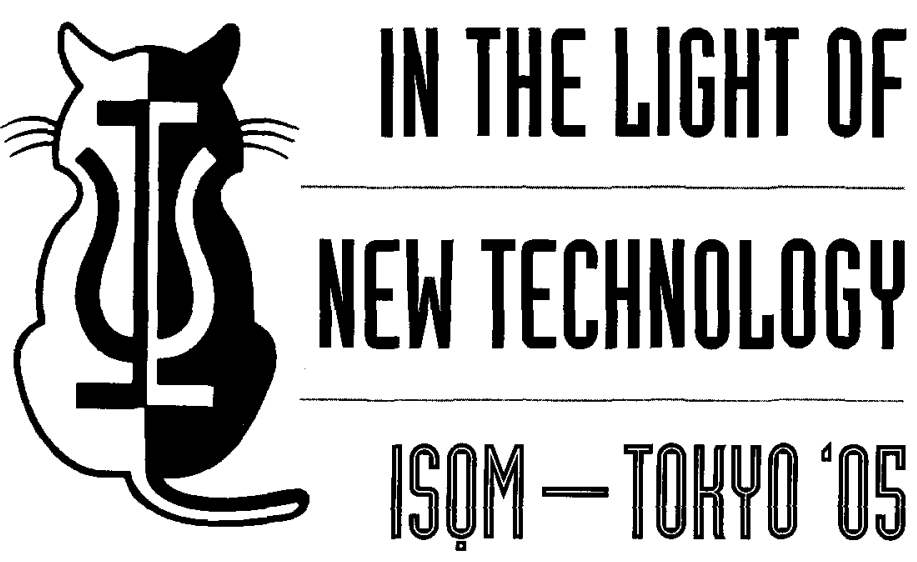

Advanced Research Laboratory

Hitachi, Ltd., Hatoyama, Saitama, Japan

22-25 August 2005

Edited by

Sachio Ishioka

Advanced Research Laboratory

Hitachi, Ltd., Japan

Kazuo Fujikawa

Institute of Quantum Science

Nihon University, Japan 


\section{Published by}

World Scientific Publishing Co. Pte. Ltd.

5 Toh Tuck Link, Singapore 596224

USA office: 27 Warren Street, Suite 401-402, Hackensack, NJ 07601

UK office: 57 Shelton Street, Covent Garden, London WC2H 9HE

\section{British Library Cataloguing-in-Publication Data}

A catalogue record for this book is available from the British Library.

\section{Proceedings of the 8th International Symposium (ISQM - Tokyo '05) FOUNDATIONS OF QUANTUM MECHANICS IN THE LIGHT OF NEW TECHNOLOGY}

Copyright (C) 2006 by World Scientific Publishing Co. Pte. Ltd.

All rights reserved. This book, or parts thereof, may not be reproduced in any form or by any means, electronic or mechanical, including photocopying, recording or any information storage and retrieval system now known or to be invented, without written permission from the Publisher.

For photocopying of material in this volume, please pay a copying fee through the Copyright Clearance Center, Inc., 222 Rosewood Drive, Danvers, MA 01923, USA. In this case permission to photocopy is not required from the publisher.

ISBN 981-256-858-1 


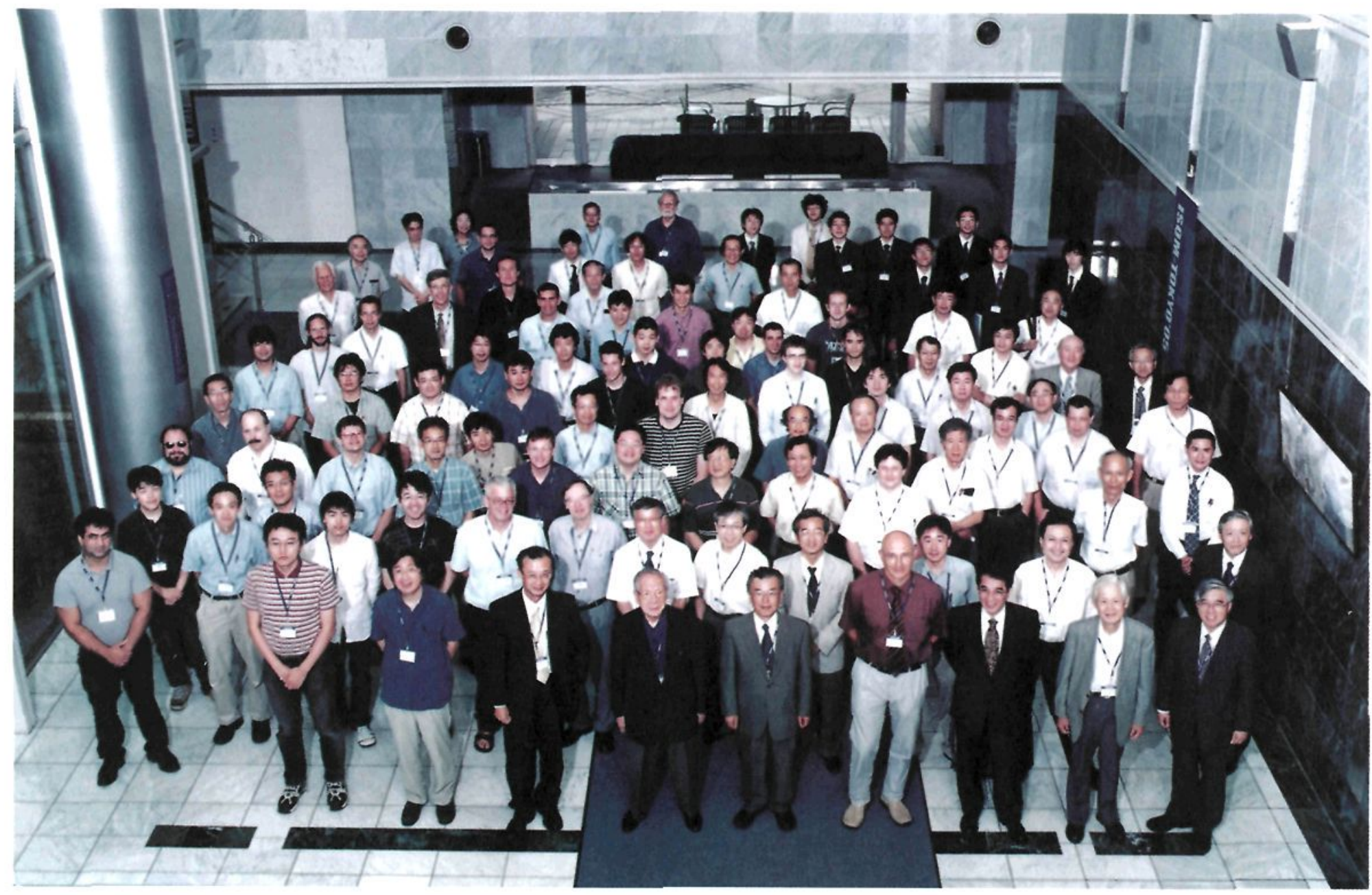

The Eighth International Symposium on Foundations of Quantum Mechanics (ISQM-Tokyo '05) 
This page is intentionally left blank 


\section{PREFACE}

The Eighth International Symposium on Foundations of Quantum Mechanics in the Light of New Technology (ISQM-TOKYO '05) was held on August 22-25, 2005 at the Advanced Research Laboratory, Hitachi, Ltd. in Hatoyama, Saitama, Japan. The symposium was organized by its own scientific committee under the auspices of the Physical Society of Japan, the Japan Society of Applied Physics, and the Advanced Research Laboratory, Hitachi, Ltd. Over 130 participants ( 23 from abroad) attended the symposium, and 30 invited oral papers, 7 contributed oral papers, and 37 poster papers were presented.

Just as in the previous seven symposia, the aim was to link the recent advances in technology with fundamental problems in quantum mechanics. It provided a unique interdisciplinary forum where scientists from different disciplines, who would otherwise never meet each other, convened to discuss basic problems of common interest in quantum science and technology from various aspects and "in the light of new technology."

Quantum Coherence, Decoherence, and Geometrical Phase was chosen as the main theme for the present symposium because of its importance in quantum science and technology. This topic was reexamined from all aspects, not only in terms of quantum computing, quantum information, and mesoscopic physics, but also in terms of the physics of precise measurement, spin related phenomena, and other fundamental problems in quantum physics. We were delighted that many active and eminent researchers in these fields accepted our invitation.

We are now very happy to offer the fruits of the symposium in the form of the proceedings to a wider audience. As shown in the table of contents, the proceedings include a special lecture on Einstein by Professor C.N. Yang, which was really well-timed with the World Year of Physics 2005, and 63 refereed papers in ten sections: quantum information and entanglement, quantum computing; quantum-dot systems, anomalous Hall effect and spin-Hall effect, spin related phenomena, superconductivity in nano-systems, novel properties of carbon nanotubes, novel properties of nano-systems, precise measurements, and fundamental problems in quantum physics. We will mention just some of the important key words here to give the flavor of the proceedings: quantum computation and communication, qubits, quantum dots, spintronics, mesoscopic spins, Berry phase, nanowires, Josephson junctions, and vortices in high-temperature superconductors. We hope that the proceedings will not only be the record of the symposium but also serve as a good reference book for experts on quantum coherence and decoherence and as an introductory book for newcomers in this field.

In conclusion, we thank the participants for their contribution to the symposium's success. Thanks are also due to all the authors who prepared manuscripts and to the referees who kindly reviewed the papers. We also thank the members of the Advisory Committee and Organizing Committee; without their invaluable assistance, the symposium would not have been a success. Finally, we would like to express our deepest gratitude to the Advanced Research Laboratory, Hitachi, Ltd. and its General Manager, Dr. Nobuyuki Osakabe, for providing us with financial support and an environment that was ideal for lively discussion. We also thank his staff members, in particular Mr. Yoshimasa Yamamoto and Ms. Maki Shinkai, for their efforts in making the symposium enjoyable as well as productive.

March 2006

Sachio Ishioka

Kazuo Fujikawa 
This page is intentionally left blank 


\section{COMMITTEES}

\section{Chair:}

H. Fukuyama, Tohoku University

\section{Advisory Committee:}

H. J. Kimble, California Institute of Technology

S. Kobayashi, Tokyo University of Agriculture and Technology

A. J. Leggett, University of Illinois at Urbana-Champaign

J. E. Mooij, Delft University of Technology

S. Nakajima, Superconductivity Research Laboratory, International Superconductivity Technology Center

N. Osakabe, Advanced Research Laboratory, Hitachi, Ltd.

F. Wilczek, Massachusetts Institute of Technology

C. N. Yang, Tsinghua University

A. Zeilinger, Vienna University

\section{Organizing Committee:}

K. Fujikawa, Nihon University

Y. Iye, University of Tokyo

N. Nagaosa, University of Tokyo

Y. A. Ono, University of Tokyo

F. Shimizu, University of Electro-Communications

H. Takayanagi, NTT Basic Research Laboratories, NTT Corporation

S. Ishioka, Advanced Research Laboratory, Hitachi, Ltd.

\section{Sponsors:}

The Physical Society of Japan

The Japan Society of Applied Physics

Advanced Research Laboratory, Hitachi, Ltd. 
This page is intentionally left blank 


\section{CONTENTS}

Preface

vii

Committees

ix

Opening Address

H. Fukuyama

1

Welcoming Address

N. Osakabe

\section{Special Lecture}

Albert Einstein: Opportunity and perception

C.N. Yang

\section{Quantum Information and Entanglement}

Quantum optics with single atoms and photons

H.J. Kimble

Quantum information system experiments using a single photon source

Quantum communication and quantum computation with entangled photons

A. Zeilinger

High-fidelity quantum teleportation and a quantum teleportation network for continuous variables

N. Takei, A. Furusawa

Long lived entangled states

H. Häffner, F. Schmidt-Kaler, W. Hänsel, C. Roos, P.O. Schmidt, M. Riebe, M. Chwalla, D. Chek-al-Kar, J. Benhelm, U.D. Rapol, T. Körber, C. Becher, R. Blatt,

Quantum non-locality using tripartite entanglement with non-orthogonal states

Quantum entanglement and wedge product 
xii

Analysis of the generation of photon pairs in periodically poled lithium niobate

$J$. Söderholm, K. Hirano, S. Mori, S. Inoue, S. Kurimura

Generation of entangled photons in a semiconductor and violation of Bell's inequality G. Oohata, R. Shimizu, K. Edamatsu

\section{Quantum Computing}

Decoherence of a Josephson junction flux qubit

Y. Nakamura, F. Yoshihara, K. Harrabi, J.S. Tsai

Spectroscopic analysis of a candidate two-qubit silicon quantum computer in the microwave regime

J. Gorman, D.G. Hasko, D.A. Williams

Berry phase detection in charge-coupled flux-qubits and the effect of decoherence

H. Nakano, S. Saito, H. Takayanagi, R. Fazio

Locally observable conditions for the successful implementation of entangling multi-qubit quantum gates

H.F. Hofmann, R. Okamoto, S. Takeuchi

State control in flux qubit circuits: manipulating optical selection rules of microwave-assisted transitions in three-level artificial atoms

Y.-X. Liu, J.Q. You, L.F. Wei, C.P. Sun, F. Nori

The effect of local structure and non-uniformity on decoherence-free states of charge qubits

T. Tanamoto, S. Fujita

Entanglement-assisted estimation of quantum channels

A. Fujiwara

Superconducting quantum bit with ferromagnetic $\pi$-Junction

T. Yamashita, S. Takahashi, S. Maekawa

Generation of macroscopic Greenberger-Horne-Zeilinger states in Josephson systems

T. Fujii, M. Nishida, N. Hatakenaka

\section{Quantum-Dot Systems}

Tunable tunnel and exchange couplings in double quantum dots

S. Tarucha, T. Hatano, M. Stopa 
Coherent transport through quantum dots

S. Katsumoto, M. Sato, H. Aikawa, Y. Iye

Electrically pumped single-photon sources towards $1.3 \mu \mathrm{m}$

X.Xu, D.A. Williams, J.D. Mar, J.R.A. Cleaver

Aharonov-Bohm-type effects in antidot arrays and their decoherence

M. Kato, H. Tanaka, A. Endo, S.Katsumoto, Y. Iye

Nonequilibrium Kondo dot connected to ferromagnetic leads

Y. Utsumi, J.Martinek, G. Schön, S. Maekawa

Full counting-statistics in a single-electron transistor in the presence of strong quantum fluctuations

Y. Utsumi

\section{Anomalous Hall Effect and Spin-Hall Effect}

Geometry and the anomalous Hall effect in ferromagnets

N.P. Ong, W.-L. Lee

Control of spin chirality, Berry phase, and anomalous Hall effect

Y. Tokura, Y.Taguchi

Quantum geometry and Hall effect in ferromagnets and semiconductors

N. Nagaosa

Spin-Hall effect in a semiconductor two-dimensional hole gas with strong spin-orbit coupling

J. Wunderlich, B. Kaestner, K. Nomura, A.H. MacDonald, J. Sinova, T. Jungwirth

Intrinsic spin Hall effect in semiconductors

S. Murakami

\section{Spin Related Phenomena}

Theory of spin transfer phenomena in magnetic metals and semiconductors A.S. Núñez, A.H. MacDonald

Spin filters of semiconductor nanostructures

T. Dietl, G. Grabecki, J. Wróbel 
xiv

Experimental study on current-driven domain wall motion

T. Ono, A. Yamaguchi, H. Tanigawa, K. Yano, S. Kasai

Magnetization reversal of ferromagnetic nano-dot by non local spin injection

Y. Otani, T. Kimura

Theory of current-driven domain wall dynamics

G. Tatara, H. Kohno, J. Shibata, E. Saitoh

Magnetic impurity states and ferromagnetic interaction in diluted magnetic semiconductors

M. Ichimura, K. Tanikawa, S. Takahashi, G. Baskaran, S. Maekawa

Geometrical effect on spin current in magnetic nano-structures

M. Ichimura, S. Takahashi, S. Maekawa

Ferromagnetism in anatase $\mathrm{TiO}_{2}$ codoped with $\mathrm{Co}$ and $\mathrm{Nb}$

T. Hitosugi, T. Shimada, T. Hasegawa, G. Kinoda, K. Inaba,

Y. Yamamoto, Y. Furubayashi, Y. Hirose

\section{Superconductivity in Nano-Systems}

Nonlinear quantum effects in nanosuperconductors

C. Carballeira, G. Teniers, V.V. Moshchalkov, A. Ceulemans

Coalescence and rearrangement of vortices in mesoscopic superconductors

A. Kanda, N. Shimizu, K. Tadano, Y. Ootuka, B.J. Baelus, F.M. Peeters,

K. Kadowaki

Superconductivity in topologically nontrivial spaces

M. Hayashi, T. Suzuki, H. Ebisawa, M. Kato, K. Kuboki

DC-SQUID ratchet using atomic point contact

Y. Ootuka, H. Miyazaki, A. Kanda

Superconducting wire network under spatially modulated magnetic field

H. Sano, A. Endo, S. Katsumoto, Y. Iye

Simple and stable control of mechanical break junction for the study of superconducting atomic point contact

H. Miyazaki, A.Kanda, Y. Ootuka, T. Yamaguchi

Critical currents in quasiperiodic pinning arrays: One-dimensional chains and Penrose lattices

V. R. Misko, S. Savel'ev, F. Nori 
Macroscopic quantum tunneling in high-Tc superconductor Josephson junctions S. Kawabata

\section{Novel Properties of Carbon Nanotubes}

Carbon nanotubes and unique transport properties: Importance of symmetry and channel number

$$
\text { T. Ando }
$$

Optical processes in single-walled carbon nanotubes threaded by a magnetic flux J. Kono, S. Zaric, J. Shaver, X. Wei, S.A. Crooker, O. Portugall,

G.L.J.A. Rikken, R.H. Hauge, R.E. Smalley

Non-equilibrium transport through a single-walled carbon nanotube with highly transparent coupling to reservoirs

P. Recher, N. Y. Kim, Y. Yamamoto

\section{Novel properties of Nano-systems}

Transport properties in low dimensional artificial lattice of gold nano-particles

S. Saito, T. Arai, H. Fukuda, D. Hisamoto, S. Kimura, and T. Onai

First principles study of dihydride-chain structures on $\mathrm{H}$-terminated Si(100) surface

Y. Suwa, M. Fujimori, S. Heike, Y. Terada, T. Hashizume

Electrical property of $\mathrm{Ag}$ nanowires fabricated on hydrogen-terminated Si(100) surface

M. Fujimori, S. Heike, T. Hashizume

Effect of environment on ionization of excited atoms embedded in a solid-state cavity M. Ando, C. Lee, S. Saito, Y. A. Ono

Development of universal virtual spectroscope for optoelectronics research:

First principles software replacing dielectric constant measurements

T. Hamada, T. Yamamoto, H. Momida, T. Uda, T. Ohno, N. Tajima,

S. Hasaka, M. Inoue, N. Kobayashi

Quantum Nernst effect

H. Nakamura, N. Hatano, R. Shirasaki 
xvi

\section{Precise Measurements}

Quantum phenomena visualized using electron waves

A. Tonomura

270

An Optical lattice clock: Ultrastable atomic clock with engineered perturbation

H. Katori, M. Takamoto, R. Higashi, F.-L. Hong

Development of Mach-Zehnder interferometer and "coherent beam steering" technique for cold neutron

K. Taketani, H. Funahashi, Y. Seki, M. Hino, M. Kitaguchi, R. Maruyama,

Y. Otake, H.M. Shimizu

Surface potential measurement by atomic force microscopy using a quartz resonator S. Heike, T. Hashizume

\section{Fundamental Problems in Quantum Physics}

Berry's phases and topological properties in the Born-Oppenheimer approximation K. Fujikawa

Self-trapping of Bose-Einstein condensates by oscillating interactions H. Saito, M. Ueda

Spinor solitons in Bose-Einstein condensates - Atomic spin transport J. Ieda

Spin decoherence in a gravitational field

H. Terashima, M. Ueda

Berry's phase of atoms with different sign of the $g$-factor in a conical rotating magnetic field observed by a time-domain atom interferometer

A. Morinaga, H. Narui, A. Monma, T. Aoki

List of participants

Author Index 